phenylalaninemia imitates the metabolic condition at a lower level but will not, even if untreated, cause mental retardation

19. The authors gratefully acknowledge the technical assistance of Miss Erika Grube, and are indebted to Dr. Hildgund Schmidt for much help during the investigation.
20. This work was supported by the Deutsche Forschungsgemeinschaft (SFB 35).

21. Requests for reprints should be addressed to: Klaus Bartholomé, M.D., 6900 Heidelberg, Universitäts-Kinderklinik, Im Neuenheimer Feld 150, Federal Republic of Germany.

22. Accepted for publication July 25, 1975
Bilirubin mitochondria ligandin oxidative phosphorylation

\title{
Ligandin Reverses Bilirubin Inhibition of Liver Mitochondrial Respiration in Vitro
}

\author{
KAZUAKI KAMISAKA, ZENAIDA GATMAITAN, CYRIL L. MOORE, AND \\ IRWIN M. ARIAS(17) \\ The Liver Research Center, Division of Gastroenterology-Liver Diseases, Department of Medicine and Department \\ of Neurology. Albert Einstein College of Medicine, Bronx. New York. USA
}

\section{Extract}

Ligandin, an abundant cytoplasmic binding protein of bilirubin and other ligands in liver cells, completely prevented the inhibitory effect of bilirubin on respiration and oxidative phosphorylation by isolated rat liver mitochondria. At equimolar concentrations of bilirubin and ligandin or human serum albumin, mitochondrial respiration was fully restored to control values. At greater ratios of bilirubin and ligandin or human serum albumin, the latter had a stronger protective effect. These studies suggest that ligandin may have a physiologic role in protecting mitochondrial systems against bilirubin toxicity.

\section{Speculation}

Some organs, such as liver, kidney, and intestine, transport various organic anions such as bilirubin and possess a soluble binding protein (ligandin) for protection of intracellular organelles. Other organs, such as the brain, lack this protective mechanism and, therefore, are more susceptible to toxicity as, for example, from bilirubin (kernicterus).

Unconjugated bilirubin produces toxic effects on mitochondrial electron transport, oxidative phosphorylation, and ultrastructure (I, II). Respiratory stimulation and uncoupling are observed at concentrations lower than those necessary to inhibit oxidative phosphorylation (I, II). Bovine or human serum albumin in vitro prevents injurious effects of bilirubin on isolated mitochondria by preferentially binding the bile pigment (10).

Ligandin is an abundant cytoplasmic protein found in parenchymal liver cells, proximal tubules of the kidney, and intestinal mucosa, and binds bilirubin and other ligands. Ligandin is postulated to be an important determinant in the transport of various endogenous and exogenous organic anions $(5,7)$.

We have studied the role of ligandin in protecting rat liver mitochondria against effects of bilirubin on respiration and oxidative phosphorylation.

\section{METHODS}

Crystalline bilirubin was obtained from Eastman Kodak (12) (E $=60 \times 10^{3}$ at $450 \mathrm{~nm}$ in chloroform), recrystallized, and stored at $0^{\circ}$ in the dark. Bilirubin solutions were freshly prepared at concentrations of $10^{-3}-10^{-4} \mathrm{M}, 0.02 \mathrm{M}$ aqueous $\mathrm{NaOH}$, and kept at $0^{\circ}$ in the dark. Human serum albumin (fraction $\mathrm{V}$, defatted) was obtained from Sigma (13) and prepared in solution at concentrations of $10^{-3}-10^{-4} \mathrm{M}$

Ligandin was purified from Sprague-Dawley rats as described previously (6). Livers were rapidly removed from male rats $(250-350 \mathrm{~g})$ after decapitation and placed in medium containing $0.25 \mathrm{M}$ sucrose, $1.0 \mathrm{mM}$ EDTA, and $10.0 \mathrm{mM}$ Tris- $\mathrm{HCl}, \mathrm{pH} 7.4$. The tissue was homogenized with approximately 4 volumes medium using a Potter-Elvehjem glass homogenizer. The homogenate was centrifuged at $800 \times g$ and the supernatant was centrifuged at $13,000 \times \mathrm{g}$. The crude mitochondrial pellet was washed twice in $0.25 \mathrm{M}$ sucrose containing $10 \mathrm{mM}$ Tris- $\mathrm{HCl}, \mathrm{pH}$ 7.4. Resuspended mitochondria were collected by centrifugation at $25,000 \times g$. All procedures were performed at $4^{\circ}$.

Respiration was studied polarographically using a Clarke oxygen electrode (14) and a specially designed chamber with a column of $1.5 \mathrm{ml}$. The respiration medium consisted of $0.25 \mathrm{M}$ sucrose, $5 \mathrm{mM} \mathrm{MgCl}_{2}, 20 \mathrm{mM}$ potassium phosphate, $10 \mathrm{mM}$ Tris- $\mathrm{HCl}, \mathrm{pH} 7.4(8)$

\section{RESULTS}

Addition of less than $20-30 \mu \mathrm{m}$ of bilirubin to the mitochondrial medium containing glutamate-malate as a substrate pair increased basal respiration (state 4 rate), increased phosphorylating respiration (state 3 rate), and, as shown in Figure 1, decreased the respiratory control index (state 3:4). At higher concentrations of bilirubin, a major decrease of state 3 respiration was observed.

In the presence of human serum albumin, bilirubin effects on state 3 and 4 respiration were reversed (Fig. 2). At an equimolar ratio of bilirubin and human serum albumin, the inhibitory effect of bilirubin almost disappeared. As the bilirubin concentration increased, the inhibitory effect of albumin was decreased but was 
still evident at a molar ratio in excess of 10:1. No difference in inhibitory effect was observed between defatted human serum albumin or highly purified fraction $\mathrm{V}$.

Ligandin also inhibited the stimulatory effect of bilirubin on respiration. With glutamate-malate as substrate, ligandin was less effective in preventing mitochondrial toxicity than was human serum albumin (Fig. 2). Similar inhibitory effects of human serum albumin and ligandin were observed using succinate-rotenone as substrate (Fig. 3). The effect of bilirubin on the respiratory system containing succinate-rotenone was less than with glutamatemalate, as described previously (11).

The reversibility of the effect of human serum albumin and ligandin on bilirubin toxicity was also examined. Mitochondria, medium, and bilirubin were incubated for 10 and $30 \mathrm{~min}$. Equimolar human serum albumin or ligandin was added to the mixture. Oxygen consumed in state 3 and 4 was recovered completely after $10 \mathrm{~min}$ of incubation. After $30 \mathrm{~min}$ of incubation, the markedly stimulated respiration was not recovered even by addition of human serum albumin or ligandin at a molar ratio exceeding 1:1.

\section{DISCUSSION}

Bilirubin injures certain cells in vitro and in vivo. It may cause severe brain damage (kernicterus) in neonates presumably because of uncoupling of mitochondrial oxidative phosphorylation and inhibition of oxygen uptake (9). Albumin prevents these effects of

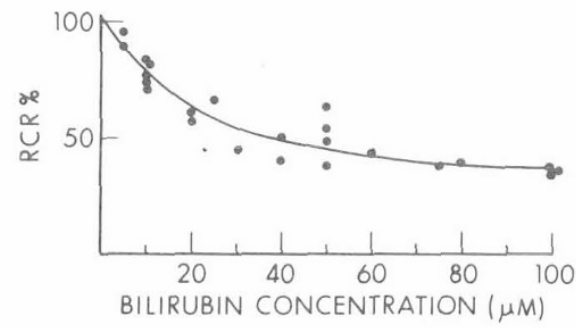

Fig. 1. The effect of bilirubin on oxygen consumption by rat liver mitochondria. Mitochondrial protein concentration was $2.0 \mathrm{mg} / \mathrm{ml}$. Substrate consisted of a mixture of $10 \mathrm{mM}$ malate and $10 \mathrm{mM}$ glutamate. State 3 respiratory rate was induced by addition of $20 \mathrm{mM}$ ADP. The respiratory control ratio $(R C R)$ is the ratio of respiratory rate in the presence of the phosphate acceptor (ADP) to the ratio in the absence of the acceptor (state 3/state 4). Since the control values varied somewhat from day to day, the data are recorded in percentages. Results are representative of six separate experiments. Each point represents results in a single cuvette.

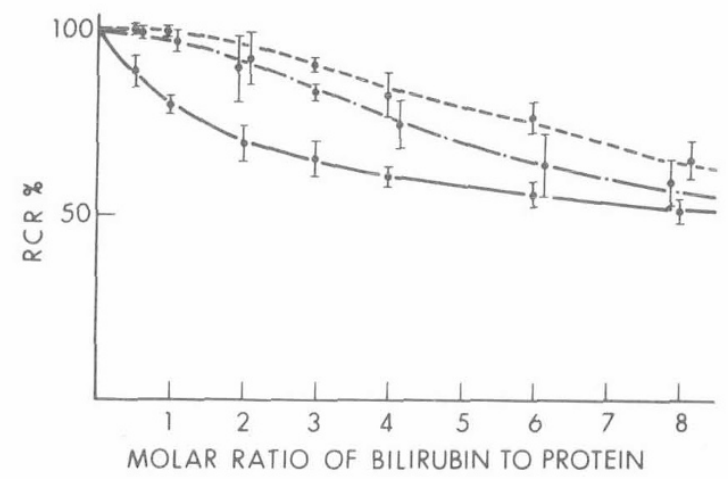

Fig. 2. Effect of human serum albumin (- - ) and ligandin (-.-) on the respiratory control ratio $(R C R)$ of glutamate $10 \mathrm{mM}$ and malate $10 \mathrm{mM}$ oxidation in the presence of bilirubin. The various concentrations of bilirubin were added to the mitochondrial medium containing $5 \mu \mathrm{m}$ of human serum albumin or ligandin. Results are expressed as mean values \pm SE from six separate experiments.

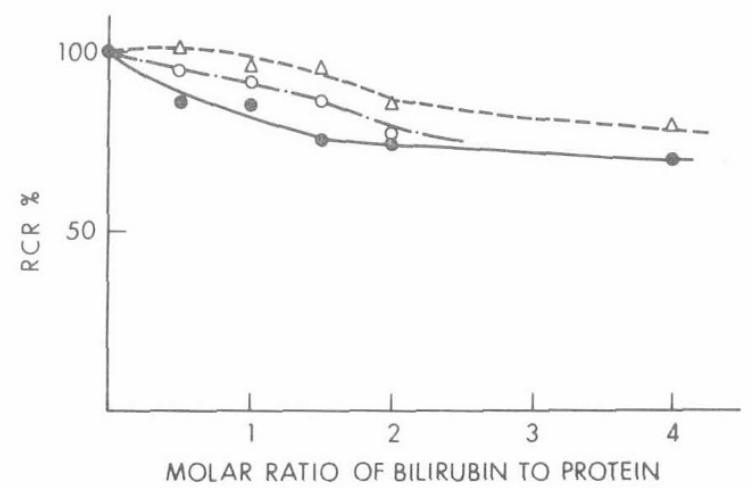

Fig. 3. Effect of human serum albumin (--) and ligandin (-.-) on the respiratory control ratio $(R C R)$ of succinate $10 \mathrm{mM}$ and rotenone $0.5 \mu \mathrm{m}$ as substrates in the presence of bilirubin. Various concentrations of bilirubin were added to the mitochondrial medium containing $5 \mu \mathrm{m}$ of human serum albumin or ligandin. Results are representative of five experiments performed in duplicate.

bilirubin on isolated mitochondria when the two are at equimolar concentration (10).

Human serum albumin and ligandin protected liver mitochondria from injury resulting from bilirubin (Fig. 2). Ligandin is a less effective inhibitor of bilirubin toxicity, which may be explained by its binding characteristics. The primary association constant for bilirubin binding to albumin is $1.4 \times 10^{8} \mathrm{M}^{-1}(3)$ and is 1 order of magnitude larger than that for bilirubin binding to ligandin, which is $5 \times 10^{7} \mathrm{M}^{-1}(5)$. Albumin also has at least two other binding sites for bilirubin as compared with a single site on ligandin $(4,5)$. Albumin should be able to remove bilirubin more effectively than is ligandin and thereby prevent bilirubin injury to mitochondria, Within the liver cell, albumin is largely in Golgi vesicles and endoplasmic reticulum, whereas ligandin, as well as bilirubin, is in the cytoplasm. This compartmentation suggests that ligandin may be the more effective soluble protein for bilirubin within the intact cell. Ligandin is abundant in liver which does not manifest mitochondrial injury by bilirubin in vivo. Ligandin is immunologically absent from brain which can manifest bilirubin toxicity (kernicterus) (2). Ligandin may protect certain organelles and functions from injurious effects of bilirubin and other potentially toxic organic anions.

\section{SUMMARY}

Ligandin as well as albumin protect against the toxic effect of bilirubin on mitochondrial oxidative phosphorylation. At higher bilirubin concentrations, human serum albumin was more effective against bilirubin toxicity. Ligandin may have a physiologically important role in intracellular protection of mitochondria from the effects of bilirubin.

\section{REFERENCES AND NOTES}

1. Cowger, M. L., Igo, R. P., and Labbe, R. F.: The mechanism of bilirubin toxicity studied with purified respiratory enzyme and tissue culture systems. Biochemistry, 4: 2763 (1965).

2. Fleischner, G., Robbins, J., and Arias, I. M.: Immunological studies of Y protein: A major cytoplasmic organic anion-binding protein in rat liver. J. Clin. Invest., 51: 677, (1972).

3. Jacobsen, J.: Binding of bilirubin to human serum albumin: Determination of the dissociation constants. Fed. Eur. Biochem. Soc. Lett., 5: 112 (1969).

4. Kamisaka, K., Listowsky, I., Betheil, J. J., and Arias, I. M.: Competitive binding of bilirubin, sulfobromophthalein, indocyanine green and other organic anions to human and bovine serum albumin. Biochim. Biophys. Acta, 365: 169 (1974).

5. Kamisaka, K., Listowsky, I., Gatmaitan, Z., and Arias, I. M.: Interactions of bilirubin and other ligands with ligandin. Biochemistry, 14: 2175 (1975).

6. Kirsch, R., Fleischner, G., Kamisaka, K., and Arias, I. M.: Structural and functional studies of ligandin, a major renal organic anion binding protein. J. Clin. Invest., 55: 1009 (1975). 
7. Levi, A. J., Gatmaitan, Z., and Arias, I. M.: Two hepatic cytoplasmic protein fractions, $\mathrm{Y}$ and $\mathrm{Z}$ and their possible role in the hepatic uptake of bilirubin, sulfobromophthalein, and other anions. J. Clin. Invest., 48: 2156 (1969).

8. Moore, C. L.: Gramicidin induced ion transport in brain mitochondria preparations. J. Neurochem.. 15: 883 (1968).

9. Odell, G. B.: Studies in kernicterus. The protein binding of bilirubin. J. Clin. Invest., 38: 823 (1959).

10. Weinbach, E. C., and Garbus, J.: Restoration by albumin of oxidative phosphorylation and related reactions. J. Biol. Chem., 24l: 169 (1966).

11. Zetterstrom, R., and Ernster, L.: Bilirubin, an uncoupler of oxidative phosphorylation in isolated mitochondria. Nature, 178: 1335 (1956).
12. Rochester, N, Y

13. St. Louis, Mo.

14. Yellow Springs Instrument Co., Yellow Springs, Ohio,

15. Dr. C. L. Moore is affiliated with the Department of Neurology. Albert Einstein College of Medicine.

16. This research was supported by United States Public Health Service Grants AM 2015, AM 5384, and AM 17702 .

17. Requests for reprints should be addressed to: Irwin M. Arias, M.D., Alber Einstein College of Medicine, Department of Medicine, 1300 Morris Park Ave., Bronx, New York 10461 (USA).

18. Accepted for publication July 25, 1975.
Hypoglycemia ketoacidosis ketotic hypoglycemia 2-methylcitrate methylmalonic aciduria propionic aciduria

\title{
Effect of 2-Methylcitrate on Citrate Metabolism: Implications for the Management of Patients with Propionic acidemia and Methylmalonic aciduria
}

\author{
SURINDER CHEEMA-DHADLI, ${ }^{(36)}$ CLIFFORD C. LEZNOFF, AND MITCHELL L. HALPERIN \\ Department of Medicine, University of Toronto, Toronto, and Department of Chemistry, York University, \\ Downsview, Ontario, Canada
}

\section{Extract}

2-Methylcitrate was tested in vitro on enzymes which interact with citrate and isocitrate. It was found to inhibit citrate synthase, aconitase, the $\mathrm{NAD}^{+}$- and $\mathrm{NADP}^{+}$-linked isocitrate dehydrogenase. This inhibition was competitive in nature except in the case of aconitase, and the $K_{i}$ for all the enzymes was in the range of 1.5-7.6 $\mathrm{mM}$. Phosphofructokinase was also inhibited by 2 -methylcitrate with $50 \%$ inhibition achieved at $1 \mathrm{mM}$. ATP-citrate lyase and acetyl-CoA carboxylase were not inhibited by this compound. 2-Methylcitrate was not a substrate for ATP-citrate lyase. Acetyl-CoA carboxylase was activated by 2 -methylcitrate with a $\mathrm{K}_{\mathrm{a}}$ of $2.8 \mathrm{mM}$. The apparent $\mathrm{K}_{\mathrm{m}}(3.3 \mathrm{mM})$ for 2-methylcitrate for the mitochondrial citrate transporter was about 10 -fold higher than the apparent $K_{m}(0.26 \mathrm{mM})$ for citrate. The tricarboxylate carrier can also be inhibited by low concentrations $(0.2 \mathrm{mM})$ of 2 methylcitrate when the concentration of citrate is close to the apparent $K_{m}$. Accumulation of 2-methylcitrate inside the mitochondrion, therefore, might lead to inhibition of enzymes in the citric acid cycle and thereby contribute to the ketogenesis and hypoglycemia seen under these conditions.

\section{Speculation}

Treatment of patients with propionic aciduria and methylmalonic aciduria with alkali therapy would be advantageous with respect to the acidemia but also would cause a more rapid exit of 2 -methylcitrate from the mitochondrion. Alkalinization with sodium citrate might be even more beneficial if this citrate could enter the liver and allow more rapid removal of 2 -methylcitrate and methylmalonate from liver mitochondria since increased cytosolic levels of these intermediates would facilitate more rapid diffusion to the extracellular space and eventual excretion in the urine. This therapy does not exclude the low protein diet and for the vitamin-responsive form of methylmalonic aciduria, $B_{12}$ treatment.

In patients with propionic and methylmalonic acidemias, 2methylcitrate has been found in the urine in substantia! quantities (1). Propionyl-CoA accumulates in liver mitochondria as a result of a deficiency of one of the enzymes required for its conversion to succinyl-CoA. 2-Methylcitrate could be formed in the mitochondrion, for example, by condensation of propionyl-CoA with oxaloacetate, despite the fact that propionyl-CoA is a poor substrate for this enzyme (26). This study was undertaken to determine whether high concentrations of 2-methylcitrate could account for any of the metabolic abnormalities found in patients with these disorders. Therefore, the effect of 2-methylcitrate was studied on the enzymes and mitochondrial transport system known to be involved in the intermediary metabolism of citrate.

The movement of citrate from the mitochondrial to the cytosolic compartment on the tricarboxylate carrier subserves several important functions in the pathway of fatty acid synthesis. It is the major pathway for two-carbon unit flow from mitochondria to cytosol (17). In addition, citrate in the cytosol could contribute to regulation of acetyl-CoA carboxylation $(9,20)$. Operation of the mitochondrial citrate transporter ( $L$-malate entry in exchange for mitochondrial citrate (4)) in conjunction with malate dehydrogenase and ATP-citrate lyase will also account for inward transport of cytosolic reducing power on NADH produced during the glycolytic sequence. Citrate is also an inhibitor of phosphofructokinase (5). More recently, an important regulatory role for mitochondrial citrate has been suggested in that this metabolite, in its free form. specifically inhibits pyruvate dehydrogenase phosphatase $(5,7)$. Regulation of the tricarboxylate carrier $(7,11,12,25)$ could be one 